\title{
EL LUGAR DE LAS REPRESENTACIONES EN LOS PROCESOS DE ELECCIÓN EDUCAIIVA. UN ANÁLLISIS SOBRE LAS ESTRATEGIAS DE LOS SECTORES MEDIOS MONTEVIDEANOS
}

\section{THE REPRESENTATION'S ROLE IN THE EDUCATIONAL PROCESS CHOICE. AN ANALYSIS OF THE STRAIEGIES OF THE MONTEVIDEO MIDDLE CLASS}

Fernanda Gutiérrez Martīnez1 I Artículo Recibido: 31 de mayo 2020 - Artículo Aceptado: 3 de diciembre 2020

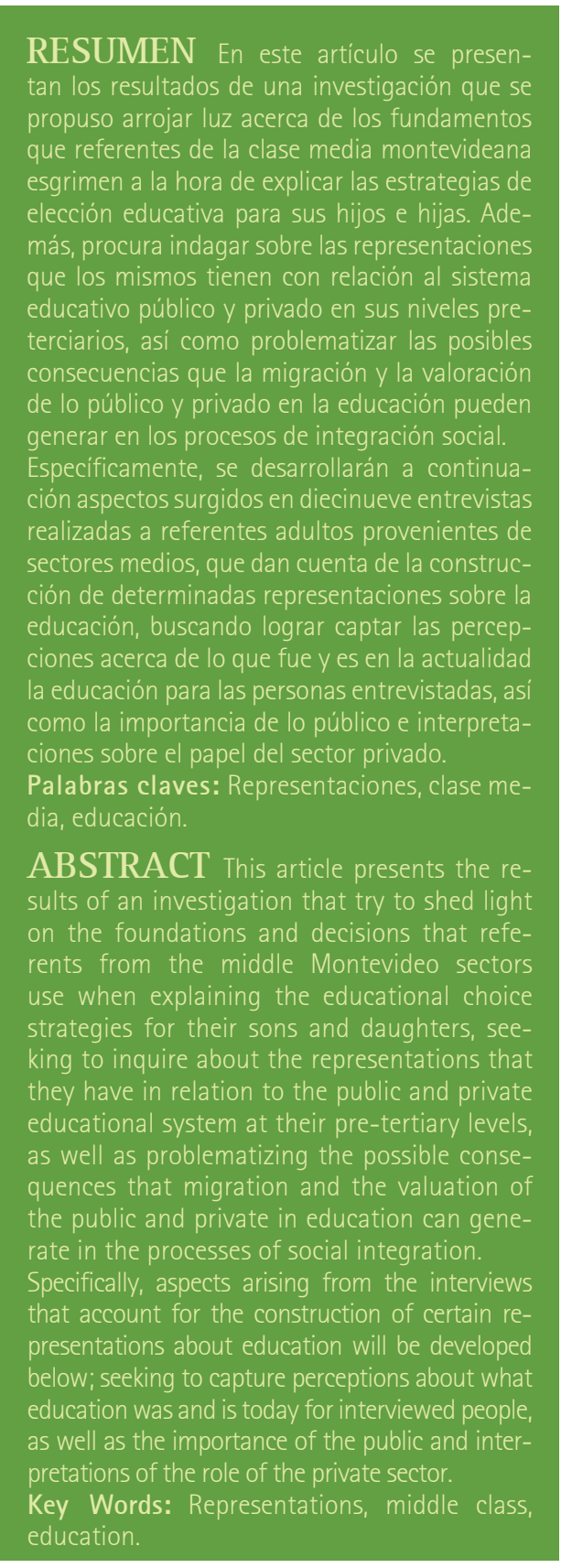

${ }^{1}$ Licenciada y Magister en Trabajo Social egresada de la Facultad de Ciencias Sociales de la Universidad de la República (Uruguay). Docente G2 del Departamento de Trabajo Social de la Facultad de Ciencias Sociales de la Universidad de la República. Correo electrónico: lic.fernandagutierrez@gmail.com

\section{INTRODUCCIÓN}

Uruguay ha sido caracterizado por diferentes denominaciones que son expresión del imaginario y de la realidad de momentos determinados de su historia. "El país modelo", de la "sociedad hiperintegrada" (Rama, 1987), de "cercanias" (Real de Azúa, 1984) y "medianías" (Real de Azúa, 1964) son adjetivos que definen a un tipo de país, a un modelo de sociedad que difiere del "país de la fragmentación estructural" (Graña y De Sierra, 2004). Cuando se mira en retrospectiva, puede apreciarse cómo la matriz social de este país incide como anclaje, pero también como motor para el cambio. La sociedad hiperintegrada, ya sea en lo que tiene de mítico como en su base real, da cuenta de un modelo de pais diferente al de hoy dadas las transformaciones en la relación Estado, mercado y sociedad.

En la búsqueda de respuestas a interrogantes vinculados a los procesos de integración social surge esta investigación ${ }^{2}$ en la que las elecciones y decisiones de los agentes juegan un papel crucial, aunque sin dejar de considerar los aspectos estructurales que tienen incidencia. Se entiende, agencia y estructura se interrelacionan y guardan interdependencia en la construcción del mundo social. Este siempre es histórico, contingente, y los individuos están produciéndolo permanentemente a través de sus prácticas, elecciones y actividades cotidianas, aunque ese mundo también los afecta e incide en su quehacer. En este sentido, los objetivos se orientaron al análisis de los fundamentos que referentes de sectores medios montevideanos esgrimen a la hora de explicar las estrategias de elección educativa para sus hijos e hijas. Además, se indaga acerca de las representaciones que los mismos tienen con relación al sistema educativo público y privado en sus niveles preterciarios, así como problematizar las posibles consecuencias que la migración ${ }^{3}$ y la valoración de lo público y privado en la educación pueden generar en los procesos de integración social.

En este artículo se presentará el resultado del análisis de los discursos surgidos en diecinueve entrevistas realizadas a veinte referentes adultos de adolescentes y jóvenes de la clase media 
De Prácticas y discursos

Universidad Nacional del Nordeste

Centro de Estudios Sociales

Año 9, Número 14, 2020, Octubre

ISSN 2250-6942

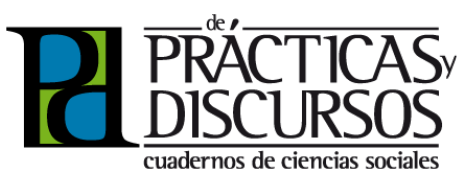

\begin{abstract}
${ }^{2}$ Esta investigación se desarrolló como requisito final de la Maestría en Trabajo Social de la Universidad de la República (UdelaR), la cual fue financiada como Proyecto de Iniciación a la Investigación por parte de la Comisión Sectorial de Investigación Científica (CSIC) de la UdelaR.
\end{abstract}

${ }^{3}$ Refiere al pasaje del sector público al sector privado de provisión de la oferta educativa.

${ }^{4}$ Tal como se desarrollará más adelante, según la perspectiva teórica desarrollada por Pierre Bourdieu, en la construcción del concepto de representaciones está imbricado el de habitus. El mismo hace referencia a la articulación entre estructura y agencia, ideas y prácticas, conocimiento y realidad. montevideana, que migraron de la enseñanza pública hacia la privada. En los discursos surgen tensiones y oscilaciones de las que se procurará dar cuenta y que se entiende, permiten comprender los movimientos en las prácticas de estos actores. Particularmente, resultó ser de interés el análisis de la transición de primaria pública hacia Educación Media Básica privada, en tanto se consideró que es relevante tener el relato de quienes optaron por lo público en determinado momento, pero que, por diferentes motivos, luego deciden migrar hacia el sector privado.

Tal como señala Martín Criado (2014), los discursos deben ser comprendidos como prácticas sociales contextualizadas que surgen a partir de determinadas condiciones de producción que los dotan de sentido social. El análisis intenta recuperar la forma en que los sujetos se adaptan a su entorno tomando decisiones estratégicas que dependen del contexto y de la posición en el espacio social en que se encuentran. Interesaba que los discursos fueran analizados a la luz de una técnica que pusiera foco en aspectos simbólicos que permitan descifrar el "decir del hacer", la justificación o argumentación de las prácticas, así como dar cuenta de representaciones que signan los modos de pensar, sentir y hacer de quienes fueron parte de la unidad de análisis de esta investigación. Entre irse y quedarse dudan los sectores medios. Lo público entraña aspectos ideológico-filosóficos que invitan a quedarse, pero algunas fallas motivan a irse y buscar lo que en este ámbito ya no se encuentra. En el fondo, el dilema parece estar centrado en este sector, entre el permanecer o el marcharse de él. Y cada uno de estos dilemas, cada una de las tensiones y oscilaciones a la hora de la decisión, la elección de cada agente, las representaciones estructuradas sobre cada sector, hacen al conjunto, repercuten en la estructura tanto como esta incidió en las tomas de decisión ${ }^{4}$.

A continuación, se presentará, específicamente, el análisis de aspectos surgidos en las entrevistas que dan cuenta de la construcción de determinadas representaciones sobre la educación, buscando lograr captar las percepciones acerca de lo que fue y es en la actualidad la educación para las personas entrevistadas, así como la importancia de lo público e interpretaciones sobre el papel del sector privado.

\section{ESTRATEGIA METODOLÓGICA}

La estrategia metodológica procuró recuperar las dimensiones micro y macro del fenómeno a indagar. Se asume que las estra- 
De Prácticas y discursos

Universidad Nacional del Nordeste

Centro de Estudios Sociales

Año 9, Número 14, 2020, Octubre

ISSN 2250-6942
El lugar de las representaciones en los procesos de elección educativa. Un análisis sobre las estrategias de los sectores medios montevideanos

The representation's role in the educational process choice. An analysis of the strategies of the montevideo middle class

\begin{abstract}
${ }^{5}$ El contenido de las entrevistas fue procesado a partir de la aplicación de la técnica para el análisis de discursos que propone Enrique Martín Criado (2010, 2014). Esta técnica, se entiende, guarda concordancia con referencias teóricas como las de Bourdieu, definidas en el marco conceptual. Interesaba que los discursos fueran analizados a la luz de una técnica que pusiera foco en aspectos simbólicos que permitan descifrar el "decir del hacer”, la justificación o argumentación de las prácticas, así como dar cuenta de representaciones que signan los modos de pensar, sentir y hacer de quienes fueron parte de la unidad de análisis de esta investigación.
\end{abstract}

${ }^{6}$ Entre 2005 y 2009 se da un aumento presupuestal de la Administración Nacional de Educación Pública (Anep) de un 71\%.

\footnotetext{
7 La “era progresista” (Garcé y Jaffé, 2004) hace referencia a las tres administraciones de gobierno frenteamplistas. El Frente Amplio ascendió al Ejecutivo por primera vez el $1^{\circ}$ de marzo de 2005 , permaneciendo durante 15 años.
}

tegias de reproducción de los sectores medios y, por consiguiente, los fundamentos que explican la migración al sector privado de provisión de la oferta educativa solo pueden explicarse analizando en forma conjunta aspectos macroestructurales y la perspectiva de los actores. En este sentido, la orientación metodológica se estructuró a partir de la adopción de datos cuantitativos obtenidos por diversas fuentes con el fin de analizar aspectos de carácter estructural que hacen al fenómeno y, por otra parte, la inclusión de métodos cualitativos que permitieran ahondar en las estrategias, representaciones y argumentos de los individuos. En cuanto a los métodos cualitativos, se apeló al análisis de los discursos $^{5}$ surgidos en diecinueve entrevistas de preguntas abiertas realizadas a veinte adultos referentes (en una entrevista participaron ambos padres) que se apoyaron en un guion temático. Se optó por la realización de entrevistas con estas características, en tanto, se constituye en una técnica de investigación cualitativa que puede orientarse al estudio de representaciones y discursos arquetípicos de grupos o colectivos (Delgado y Gutiérrez, 1999; Valles, 2000).

La unidad de análisis se conformó por los referentes adultos de adolescentes o jóvenes que se encontraban cursando al momento de la entrevista o ya habian cursado Educación Media Básica en el sector privado y que asistieron en el Nivel Primario a enseñanza pública. Fueron consideradas las decisiones tomadas a partir de 2010, en un marco de crecimiento económico, en el que aumentan las posibilidades reales de los sectores medios de optar por el sector privado de provisión de la oferta educativa, así como en el contexto de un gobierno progresista que venía destinando mayores recursos económicos a la educación públi$\mathrm{ca}^{6}$. A su vez, esta definición temporal se establece a modo de dar un plazo a las innovaciones que desde el gobierno pretendian implementarse en materia de educación (ya que la "era progresista"7 se inició en Uruguay, en marzo de 2005).

Para efectuar la selección de los hogares a los que se les aplicó la entrevista, se tomó en consideración la clasificación de escuelas realizada a partir del Relevamiento del Perfil Sociocultural Ilevado adelante por la Administración Nacional de Educación Pública (Anep) en 2015. Tal clasificación fue realizada a partir de la construcción del Índice de Características Socioculturales (ICSC), el cual considera tres dimensiones de los hogares: el nivel educativo, el socioeconómico y el grado de integración social. Ello permitió ordenar a los centros desde su perfil sociocultural más desfavorable al más favorable. 
De Prácticas y discursos

Universidad Nacional del Nordeste

Centro de Estudios Sociales

Año 9, Número 14, 2020, Octubre

ISSN 2250-6942
${ }^{8}$ Cabe precisar que estos quintiles de clasificación de la población que asiste a las escuelas públicas montevideanas, en función de su perfil sociocultural, no se corresponden con la clasificación por quintiles de ingresos a nivel de la población uruguaya. Esto quiere decir que un/a niño/a puede asistir a una escuela considerada de quintil 5 y no pertenecer a este quintil de ingresos.
A partir de estas consideraciones, la Anep agrupó las escuelas en quintiles, según los datos que arrojó el ICSC, estableciendo diferentes niveles de Requerimiento prioritario, siendo el nivel 1 (Quintil 1) el que registra el 20\% de las escuelas con mayores indices de deprivación, mientras que el Requerimiento 5 (Quintil 5) agrupa a las escuelas de mejor contexto (Anep, 2015).

Al momento de trabajar en el diseño de la estrategia metodológica, se partió de la premisa que las escuelas de mejor contexto (Quintil 5) serian las que concentrarian mayor proporción de niños provenientes de hogares de clase media, con posibilidades económicas de poder migrar a la enseñanza privada. Por consiguiente, se definió la realización de entrevistas a referentes adultos de adolescentes y jóvenes que egresaron de escuelas públicas en este nivel de clasificación, entre 2010 y 2019, hacia el sector privado de provisión de la enseñanza media.

Al aplicar una técnica de investigación cualitativa, la muestra fue definida por el principio de representación socioestructural y no por la aleatorización definida a nivel estadístico (Valles, 2000). A su vez, se apeló a los criterios de accesibilidad y heterogeneidad para llevar adelante el trabajo de campo.

Con relación a la accesibilidad, se estableció contacto con los entrevistados a través de las direcciones de las escuelas predefinidas (Quintil 5 según ICSC), docentes, así como por el contacto con actores de instituciones cercanas al contexto escolar y redes de proximidad.

En cuanto a la heterogeneidad, se procuró abarcar la mayor diversidad de situaciones posibles. Tal como señala Valles (2000), lo que interesa en la definición de una muestra cualitativa es la profundidad en el conocimiento del objeto de estudio, no siendo una prioridad la cantidad de unidades a estudiar. Cada unidad da cuenta de una posición diferencial que ocupa en la estructura social. La intención fue captar la heterogeneidad estructural del objeto de estudio, y los ejes que definieron tal heterogeneidad fueron: el eje socioeconómico, el eje espacial y el eje temporal. Estas delimitaciones conceptuales operaron como una suerte de "filtro" que condujo al grupo de entrevistados.

El eje temporal definido en el objeto de estudio responde al período en el que se concreta la migración del sector público al sector privado. Se definió que la misma debió haberse efectuado a partir de 2010 hasta 2019, tal como se indicó anteriormente; en tanto interesa analizar este proceso en el marco de gobiernos de carácter progresista, en un contexto caracterizado por mejo- 
De Prácticas y discursos

Universidad Nacional del Nordeste

Centro de Estudios Sociales

Año 9, Número 14, 2020, Octubre

ISSN 2250-6942
El lugar de las representaciones en los procesos de elección educativa. Un análisis sobre las estrategias de los sectores medios montevideanos

The representation's role in the educational process choice. An analysis of the strategies of the montevideo middle class

\begin{abstract}
${ }^{9}$ Los centros comunales son dependencias descentralizadas de la Intendencia de Montevideo, encargadas de la gestión administrativa, de trámites, denuncias, solicitudes y servicios que corresponden a una determinada delimitación territorial. Montevideo se divide en $18 \mathrm{CCZ}$, que son parte a su vez de 8 municipios. Los municipios se configuraron como el tercer nivel de gobierno y administración que se agrega a los tradicionales niveles de gobierno nacional y departamental. Cada municipio tiene una circunscripción territorial urbana y suburbana que debe conformar una unidad, con personalidad social y cultural, con intereses comunes que justifiquen la existencia de estructuras políticas representativas y que faciliten la participación ciudadana.
\end{abstract}

ras en el mercado de trabajo y por el crecimiento económico, en el que se realiza un aumento del GPS, y en particular del gasto público en educación. Se marca como inicio el 2010, en tanto comienza el segundo gobierno frenteamplista, entendiendo que se hace interesante el análisis de este proceso luego de la instrumentación de lineamientos políticos e inicio de una etapa de transformaciones en el marco de la agenda progresista.

Por su parte, el eje socioeconómico es considerado a partir de la clasificación de las escuelas. Las escuelas públicas han sido clasificadas en función de las características socioculturales de su población, considerándose a efectos de esta investigación las escuelas con ICSC 5, por entenderse que agrupan en mayor proporción a niños provenientes de las clases medias, tal como fuera dicho antes.

En cuanto a la definición del eje espacial, cabe decir que se procuró captar la heterogeneidad socioeconómica sobre la que se asientan las escuelas a nivel territorial. Más allá de que todas son clasificadas con un ICSC 5, las escuelas se ubican en zonas diferentes, con entornos que dan cuenta de distintos porcentajes de población en situación de pobreza y no pobreza.

Es por ello que se realizó una selección proporcional de las escuelas ICSC 5 según la distribución territorial de la pobreza en Montevideo, de acuerdo al Informe del INE de Estimación de la Pobreza por el método de Ingreso para 2015. Cabe señalar que se toman los datos surgidos de la Encuesta Continua de Hogares (ECH) de ese año, a efectos de que exista coincidencia temporal entre los datos vinculados a la distribución espacial de los hogares pobres en Montevideo y los que surgen del último Relevamiento de características socioculturales realizado por la Anep, a partir del cual se clasifican las escuelas en función del ICSC. La distribución de la pobreza en Montevideo es considerada de acuerdo al corte territorial definido por los Centros Comunales Zonales (CCZ) ${ }^{9}$, por representar una zona geográfica más reducida y de características más homogéneas que los municipios. A efectos prácticos, decide agruparse para esta investigación a los CCZ en tres grandes grupos: aquellos que presentan un bajo nivel de pobreza (entre 0.5 y 2.4\%), los que presentan un nivel intermedio (entre 2.5 y $13.4 \%$ ) y los que tienen un alto nivel de pobreza (13.5\% o más). Así, Montevideo queda dividido en tres grandes zonas: de alto, medio y bajo nivel de pobreza. Al analizar la concentración de las escuelas de Quintil 5, surge que el $74 \%$ se ubica en la zona con bajo nivel de pobreza, 16\% en la de nivel 
De Prácticas y discursos

Universidad Nacional del Nordeste

Centro de Estudios Sociales

Año 9, Número 14, 2020, Octubre

ISSN 2250-6942

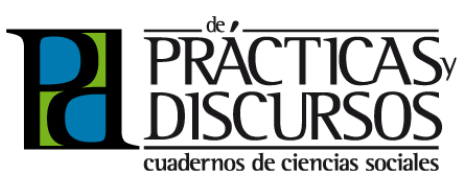

medio y $10 \%$ en la que concentran un alto nivel de pobreza. Es entonces que se decide realizar las entrevistas tomando en cuenta esta distribución, en tanto se contemplan aspectos socioeconómicos y de distribución espacial. Si bien operó la saturación teórica para definir el límite de entrevistas a realizar, se procuró que las mismas mantuvieran una distribución proporcional con relación a la concentración espacial de las escuelas.

\section{PERFIL DE LAS PERSONAS ENTREVISTADAS}

Interesa describir algunas características generales de las personas entrevistadas. Se entrevistó a catorce mujeres y seis varones, ya que en una de las entrevistas participaron ambos referentes adultos del joven que hizo la transición a analizar. Sus edades varian entre los 35 y los 58 años de edad.

Todas las personas entrevistadas desempeñan tareas laborales fuera del hogar. Son en su amplia mayoría profesionales egresados de estudios universitarios o terciarios (17 de 20), varios de ellos con estudios de posgrado. La inserción laboral se da fundamentalmente en relación de dependencia en el sector público (14), existiendo dos casos de dependencia en el sector privado, dos que mantienen empleos en ambos sectores, mientras que una entrevistada se dedica a la actividad comercial contando con local propio y un entrevistado se encuentra desocupado, teniendo como principal actividad la de estudiante universitario. En cuanto a la ocupación, dieciséis desempeñan tareas profesionales, dos tareas técnico-administrativas y dos actividad comercial (considerando la actividad que desarrollaba quien estaba desocupado).

En cuanto a la zona de residencia, coincide en la mayoría de los casos con la ubicación de la escuela, es decir, gran parte de las personas entrevistadas residen en el mismo barrio en que se encuentra el centro educativo que eligieron para que sus hijos e hijas cursaran la primaria. Solo se presenta un caso en el que no se da esta coincidencia, aunque la escuela elegida se encontraba próxima al lugar de trabajo. En este sentido, la mayor parte de las personas entrevistadas (17) residen en zonas de nivel bajo o medio de pobreza.

La trayectoria educativa de las personas entrevistadas es una de las preguntas con la que en general se inician las entrevistas. Es de destacar que todas tuvieron alguna experiencia en el ámbito público si consideramos los tres niveles: Primaria, Secundaria y Terciaria o Universidad. Ninguno de los entrevistados cursó Edu- 
De Prácticas y discursos

Universidad Nacional del Nordeste

Centro de Estudios Sociales

Año 9, Número 14, 2020, octubre

ISSN 2250-6942
El lugar de las representaciones en los procesos de elección educativa.

Un análisis sobre las estrategias de los sectores medios montevideanos

The representation's role in the educational process choice. An analysis of the strategies of the montevideo middle class

cación Técnica (UTU) en enseñanza Media. Es de relevancia que todas las que hicieron estudios terciarios o universitarios (18) cursaron en el sector público. Cuando esto lo limitamos a los primeros dos niveles, la mayoría (16) da cuenta de haber cursado al menos uno en el ámbito público, mientras que solo cuatro hicieron estos trayectos exclusivamente en el sector privado.

\section{ACERCA DE LAS REPRESENTACIONES}

Las representaciones se constituyen en principios de clasificación, en esquemas de percepción compartidos, que se producen en función de la posición ocupada en el espacio social y a partir del conocimiento práctico, incidiendo a su vez en el ejercicio de nuevas formas de hacer. Estas maneras de pensar y crear la realidad social están constituidas por elementos de carácter simbólico, ya que no son solo formas de adquirir y reproducir el conocimiento, sino que tienen la capacidad de dotar de sentido a la realidad social.

En el proceso de análisis discursivo se detectan percepciones e interpretaciones compartidas acerca de la educación, ya sea en lo que es como en lo que se considera debe ser, así como con relación a la carga valorativa y a lo que simboliza para este grupo de personas entrevistadas.

Es el espacio social, en tanto realidad invisible e intangible, el que organiza las prácticas y las representaciones de los agentes. Estas se construyen a través de las interacciones y del conocimiento práctico de los sujetos, estructurándose a partir de ello esquemas de pensamiento, percepción e interpretación del mundo social. En este sentido, Bourdieu (1988: 477) señala,

entre las condiciones de existencia y las prácticas o las representaciones, se interpone la actividad estructurante de los agentes que, lejos de reaccionar mecánicamente a unos estímulos, responden a los llamados de un mundo cuyo sentido ellos mismos han contribuido a producir.

Así, las representaciones se constituyen en principios de clasificación, en esquemas de percepción compartidos, que se producen de acuerdo con la posición ocupada en el espacio social y a partir del conocimiento práctico, incidiendo a su vez en el ejercicio de nuevas prácticas. Estas formas de pensar y crear la realidad social están constituidas por elementos de carácter simbólico, ya que no son solo formas de adquirir y reproducir el 
De Prácticas y discursos

Universidad Nacional del Nordeste

Centro de Estudios Sociales

Año 9, Número 14, 2020, Octubre

ISSN 2250-6942

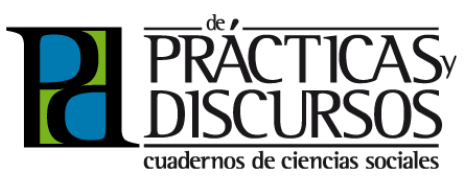

\begin{abstract}
${ }^{10}$ Los habitus asociados a una clase particular de condiciones de existencia son "sistemas de disposiciones duraderas y transferibles, estructuras estructuradas predispuestas a funcionar como estructuras estructurantes, es decir, como principios generadores y organizadores de prácticas y de representaciones que pueden ser objetivamente adaptadas a su meta sin suponer el propósito consciente de ciertos fines ni el dominio expreso de las operaciones necesarias para alcanzarlos, objetivamente 'reguladas' y 'regulares' sin ser para nada el producto de la obediencia a determinadas reglas, $y$, por todo ello, colectivamente orquestadas sin ser el producto de la acción organizadora de un director de orquesta" (Bourdieu, 2007: 86).
\end{abstract}

conocimiento, sino que tienen la capacidad de dotar de sentido a la realidad social (Bourdieu, 1988).

En la construcción del concepto de representaciones puede decirse que está imbricado el de habitus ${ }^{10}$. El mismo articula estructura y agencia, ideas y prácticas, conocimiento y realidad. El espacio de las posiciones sociales se constituye en el espacio de las tomas de posición a través del de las disposiciones, es decir, de los habitus. Una de las funciones de este concepto radica en dar cuenta de la unidad que liga las prácticas y los bienes de un agente o de una clase de agentes.

Las distintas posiciones en el espacio social, dada la desigual distribución del capital, se traducen en diferencias de disposición y de tomas de posición y a través de los habitus, en prácticas distintas. Los discursos son a su vez prácticas. Los mismos están contextualizados en un momento y lugar dados, y son expresión de esquemas de pensamiento y percepción internalizados. Su análisis en el marco de esta investigación se propuso captar la lógica de una realidad empirica, históricamente situada, con la intención de dar cuenta de representaciones, de estrategias de reproducción y del conjunto de disposiciones de un determinado grupo de agentes que comparten una posición próxima en el espacio social. Múltiples aspectos surgieron en los discursos que permitieron hilvanar y reconstruir en cierto modo la percepción, las formas de sentir, hacer y pensar de las personas entrevistadas con relación al objeto de investigación.

\section{ENTRE LA NOSTALGIA Y LA REALIDAD: LA AÑORANZA DE LA EDUCACIÓN DE OTRA ÉPOCA}

Un aspecto recurrente en las apreciaciones expresadas por las personas entrevistadas refiere a la nostalgia por un tipo de educación que entienden ya no se desarrolla de la misma manera en el presente. En su mayoría, los referentes entrevistados consideran que la educación pública en la actualidad padece de un déficit de calidad respecto a la que ellos transitaron décadas atrás. Sin embargo, algunos identifican que aún existen ciertos vestigios de esa educación pública de otra época, que pueden encontrarse en centros educativos específicos, ubicados en determinadas zonas de la ciudad, y que el mantenimiento de la calidad en esos casos responde más que nada a las características del enclave territorial del centro. 
De Prácticas y discursos

Universidad Nacional del Nordeste

Centro de Estudios Sociales

Año 9, Número 14, 2020, Octubre

ISSN 2250-6942
El lugar de las representaciones en los procesos de elección educativa.

Un análisis sobre las estrategias de los sectores medios montevideanos

The representation's role in the educational process choice. An analysis of the strategies of the montevideo middle class

Hay escuelas que todavía siguen siendo aquellas escuelas de cuando yo iba a la escuela, la Berro, la Bélgica, la Grecia, la Brasil, que todavía no cayeron en esa desgracia de que son un comedor o un lugar de contención para que los gurises no estén en el cante o estén en el carrito. Pero creo que vamos camino a eso, y cada vez más en la medida de que el presupuesto de la educación sea cuestionado... No querés invertir en educación, bueno, ¿cuánto más vas a tener que invertir en otras cosas, por no invertir en educación? (Entrevista 9)

La integración social es un aspecto recurrente en los discursos, en especial al hacer referencia a la escuela. Si bien la participación en un espacio público en el que confluyen niños y niñas provenientes de diferentes sectores socioeconómicos es un aspecto que en el presente continúa siendo valorado por los referentes adultos y considerado a la hora de optar por un determinado centro educativo, muchas de las personas entrevistadas manifiestan que, al cotejar con el pasado o al comparar experiencias transitadas en el interior del pais con la realidad montevideana, perciben una disminución en las posibilidades de interacción real de los diferentes estratos socioeconómicos en la actualidad. En este sentido, surge en varios casos que la opción por lo privado aparece al evaluar que la educación pública ha sufrido una serie de cambios que han ido en detrimento de la calidad que supo ofrecer en otra época. También se indica que si la propuesta educativa pública fuera diferente, si experimentara una serie de cambios que la tornara "atractiva", no dudarian en mantener a sus hijos e hijas en este sector de provisión durante toda su trayectoria educativa

Si yo viviera en otro país y la educación pública es lo que era cuando yo era más chica, o antes incluso, no lo pienso dos veces. No es que va a un colegio privado porque quiero que se codee, no es que me sobre. (Entrevista 12)

No se evidencia en los discursos la existencia de representaciones sobre lo privado que signen prácticas y modos de vida promercado, sino que, por el contrario, se da cuenta de una positiva valoración de la participación en ámbitos públicos e incluso de discursos que refieren a posicionamientos proestatales. En la mayoría de las entrevistas, la opción por lo privado se señala como una alternativa ante carencias o fallas visualizadas en lo público y no como una alternativa deseada y valorada en sí misma. En Uruguay, históricamente la presencia 
De Prácticas y discursos

Universidad Nacional del Nordeste

Centro de Estudios Sociales

Año 9, Número 14, 2020, Octubre

ISSN 2250-6942

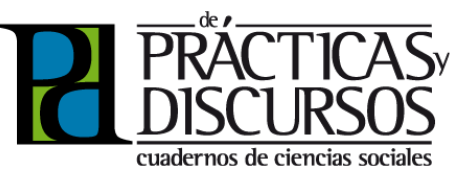

del Estado en la trayectoria biográfica de las personas ha sido significativa por el fuerte peso, la dimensión y el alcance de su intervención. En la educación esto se expresa con particularidad. La primacía estatal respecto al sector privado se mantiene al dia de hoy, aun cuando este último ha ganado terreno en esta arena política. Los referentes adultos entrevistados transitaron su educación en un momento de mayor preeminencia del sector público y se entiende, esa configuración estructural ha aportado en la construcción de las representaciones estructuradas sobre la educación.

\section{LO QUE TIENEN PARA DECIR DE LOS DOCENTES}

En la construcción de representaciones sobre la educación, los maestros y profesores tienen un lugar protagónico por ser figuras centrales y esenciales de la institucionalidad educativa. Las menciones al colectivo docente surgen, de manera casi inevitable, en todas las entrevistas, pese a que no hubo una pregunta específica sobre el mismo. Las personas entrevistadas dan cuenta en sus discursos de la imagen, de las valoraciones y opiniones con relación a los/as docentes. Por ser parte central del sistema educativo, las representaciones que se hagan sobre el cuerpo docente incidirán inevitablemente en las estructuradas sobre el sistema educativo en su conjunto.

Tal como señala Dubet (2007), la tarea docente se transforma a la par de la institución escolar. La vocación que caracterizó inicialmente a la docencia se convierte en profesión con la finalidad de superar las adversidades de la relación pedagógica. La autoridad carismática del docente, legitimado por los principios superiores que representa, entra en crisis junto al proyecto educativo moderno que fue exitoso hasta la primera mitad del siglo XX.

Los discursos de las personas entrevistadas reflejan esta crisis. Dan cuenta también de tensiones. Las opiniones oscilan entre la intención de destacar la labor docente y la crítica a estos profesionales. La idea sobre la vocación está presente. Este valor sagrado legitimador de la figura del docente aparece en forma explícita e implícita en los discursos y se mueve entre el reconocimiento y el desprestigio, entre la comprensión acerca de las condiciones del sistema y lo injustificable. 
De Prácticas y discursos

Universidad Nacional del Nordeste

Centro de Estudios Sociales

Año 9, Número 14, 2020, Octubre

ISSN 2250-6942
El lugar de las representaciones en los procesos de elección educativa.

Un análisis sobre las estrategias de los sectores medios montevideanos

The representation's role in the educational process choice. An analysis of the strategies of the montevideo middle class

Yo no sé por qué estudian, porque no hay como otro valor en la educación, si no te gusta educar. (Entrevista 2)

Qué es lo que hoy veo en los docentes, es falta de vocación [...] No sé si es el sistema que llevó a esa desidia. (Entrevista 8)

Estas tensiones que reflejan un tipo de institución y, en particular, un modelo de docente en crisis, son percibidas de forma similar entre el ámbito público y el privado. En general, las personas entrevistadas plantean que los profesores "son los mismos". De todos modos, si hay referencias a los condicionamientos que cada sector impone en la modalidad y en la capacidad de desarrollar la labor docente.

Las apreciaciones vertidas revelan la representación de un sector público caracterizado por paros docentes, faltas reiteradas de los mismos, dificultad para cubrir la totalidad de las vacantes, carencia de figuras coordinadoras, mientras que, en el privado, a pesar de que "son los mismos", las personas entrevistadas no identifican tantas faltas, paros o ausencia de asignaturas. Se percibe que en este sector los docentes tienen otro tipo de controles, de exigencias, de requerimientos. Aunque se marcan estas diferencias, las mismas no siempre indican que lo negativo está en lo público y lo positivo en lo privado, ya que también surge la idea de que los profesores y maestros más formados están en el ámbito público, a la vez que están en ese sector los que se identifican con "mayor vocación" por las adversidades que enfrentan, mientras que en el sector privado se aprecia que estos están muchas veces limitados en su libertad de ejercicio profesional, o que no necesariamente son docentes titulados.

por cuentos de gente conocida, de que los hijos no sé, están a mitad de año y todavía no tienen profesor asignado, el profesor empieza después, o bueno, que hay siempre problemas con las faltas de los profesores por temas sindicales, o por lo que fuera. Que están en todo su derecho, pero bueno, eso también resiente la formación de los alumnos. (Entrevista 3)

Yo como médico lo he vivido en el consultorio, ;ah!, certificame. Los profesores me dicen certificame solo en el público porque al privado tengo que ir igual, pierdo tanta plata, en el público igual no me descuentan. Esas cosas las vengo viendo hace años. (Entrevista 12) 
De Prácticas y discursos

Universidad Nacional del Nordeste

Centro de Estudios Sociales

Año 9, Número 14, 2020, Octubre

ISSN 2250-6942

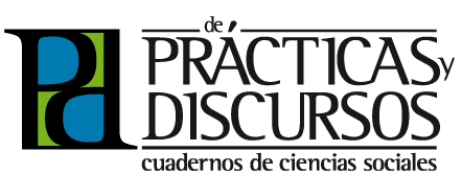

Las tensiones en los discursos acerca de lo que los docentes representan son, a su vez, reflejo de un proceso de incertidumbre y cambio, de una institucionalidad en declive, de un colectivo en crisis, de una legitimidad que se desvanece. Ante la crisis de vocación, los docentes están llamados a trabajar sobre su formación, señala Dubet (2007). La profesionalización parece ser la vía para superar los nuevos retos que enfrentan en el aula y, a la vez, es quizás el camino para la relegitimación. Las referencias a las falencias del sistema, a los obstáculos que este impone a los docentes para su formación permanente, así como la estructura de grados y de elección de centros, son aspectos que no estuvieron ausentes en los discursos y que reflejan las tensiones propias del sistema, los dilemas que enfrenta.

Aunque la figura del/la maestro/a es en general resaltada y entendida como un valor relevante en el funcionamiento de la institución escolar, también aparecen críticas. Los discursos traen referencias a los docentes que están signadas por la tensión entre la valoración de su rol, tarea, compromiso, vocación y, por otra parte, por el cuestionamiento y crítica a su desempeño, al ejercicio profesional, con referencias acerca de la falta de compromiso y vocación. Si bien de los discursos surge que el mayor peso de la crítica recae sobre los docentes de secundaria, los maestros no dejan de estar en un lugar controvertido.

Percepciones sobre la educación pública

A diferencia de las apreciaciones sobre los docentes, las percepciones sobre fortalezas y debilidades de la educación pública uruguaya sí son solicitadas expresamente en el marco de la entrevista. Con esta pregunta se procuró ahondar en las representaciones que específicamente los entrevistados tienen sobre el sistema educativo público en general, y de cada uno de sus niveles en particular.

Al referir a las debilidades, los aspectos mencionados son expresión del proceso de crisis y transformación de la institución educativa. Esa crisis no responde solo a la dificultad de adaptación a un entorno que cambia, sino que, tal como señala Dubet (2007), es una crisis del propio proceso de socialización, fundamentalmente, del trabajo sobre el otro.

En este sentido, las referencias a formatos educativos que no responden a la situación actual de las familias, propuestas que en contenido y forma no son seductoras para niños y adolescentes de esta época, programas que no se aproximan a las necesi- 
dades formativas de hoy en día, son algunas de las apreciaciones vertidas por los entrevistados.

respecto a la escuela, a primaria, creo que tenemos hoy una escuela que fue pensada para el siglo pasado. ¿Por qué digo esto? Porque tenemos una escuela que sigue siendo de cuatro horas en general, que no se adapta a las dinámicas familiares ni de trabajo de hoy en día. Desde hace unos cuarenta años, el mercado de trabajo ha cambiado drásticamente con la incorporación de la mujer. Hoy, las mujeres no trabajan a tiempo parcial, trabajan jornadas muy extensas y es impensable que la mayor parte de las familias puedan subsistir sin la incorporación de la mujer al mercado de trabajo. Entonces, con una escuela de cuatro horas, ¿cómo piensa el Estado que las familias pueden arreglárselas? [...] Tenés otras propuestas en primaria, las escuelas de tiempo completo o las experiencias de tiempo extendido que son muy interesantes, pero que de todas maneras muestran que está todo estructurado para una escuela de cuatro horas. Las escuelas de tiempo completo ofrecen cuatro horas de clase y luego no mucho más. Se convierten en un lugar donde quedan los chiquilines para ser cuidados. No hay una propuesta pensada para ocho horas. Tienen comedor y alguna otra actividad, pero no hay una propuesta sólida y bien armada para esas ocho horas. El tiempo extendido que se aplica en algunas escuelas es interesante, pero también está mal pensado. Porque está el módulo de cuatro horas con las maestras y después los chiquilines quedan ahí, sin mucha cosa para hacer, porque no está pensado. Hay algunos talleres, pero queda igualmente mucho tiempo sin actividad. V fue unos años a la escuela pública $Y$, que tiene tiempo extendido, y la mayoría de los días tenían clase cuatro horas, almorzaban y después no tenían más nada, quedaban sin actividad hasta la salida. (Entrevista 11)

En este ejercicio reflexivo y discursivo, las diferencias entre primaria y secundaria vuelven a estar presentes. De algún modo, los entrevistados comienzan a dejar entrever posibles motivaciones y fundamentos de las prácticas en materia de elección escolar. Por sus características, la educación primaria logra una contención que no logra la secundaria, según señalan las personas entrevistadas. El formato sobre el que está asentada la formación a nivel secundario no contribuye al seguimiento, apoyo y contención de los estudiantes. En cambio, en primaria, donde hay un solo adulto referente, las 
De Prácticas y discursos

Universidad Nacional del Nordeste

Centro de Estudios Sociales

Año 9, Número 14, 2020, Octubre

ISSN 2250-6942

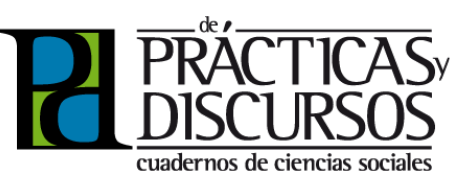

11 Organizaciones integradas por familias y vecinos que apoyan la gestión de la Dirección escolar cooperando en múltiples aspectos excepto los del ámbito pedagógico. posibilidades de dar seguimiento, de tener un conocimiento cabal sobre habilidades y competencias, de conocer el proceso educativo y la situación particular de los alumnos aumenta.

De todos modos, hay debilidades identificadas que atraviesan ambos niveles y que refieren más al formato, al "programa" en el sentido que lo define Dubet (2007). Dentro de las debilidades se identifica el carácter normalizador de la educación, lo cual le da cierta rigidez a la estructura educativa, impidiendo o dificultando la valoración de otros atributos de los estudiantes más allá de los esperados por la institución. De esta forma, quienes posean los conocimientos y habilidades valorados por la escuela tendrán mucha más facilidad para triunfar en ella (Bourdieu y Passeron, 1977).

Por otra parte, lo entendido como "extracurricular" se visualiza débil o nulo en la propuesta educativa pública. Lo que existe es por el apoyo de las Comisiones de Fomento"11, o por la impronta de determinada dirección o equipo docente, pero no porque sea un lineamiento definido a nivel de la política para ser ejecutado en todos los centros educativos del pais.

En este sentido, surge que una de las principales debilidades que presenta la educación pública a nivel de primaria y secundaria refiere a la "rigidez" institucional, a la dificultad que impone el sistema para poder procesar cambios y producir nuevas propuestas. Si bien se reconoce que esa rigidez confiere también garantías en los procesos, se traduce muchas veces en imposibilidad, en impotencia práctica, en poco margen de maniobra. Esta falta de flexibilidad impacta en la gestión, en las posibilidades de hacer y en los tiempos de ese poder hacer.

También surge la falta de recursos y condiciones apropiadas para llevar a cabo un adecuado proceso educativo. Los docentes aparecen resaltados en su rol por tener que compensar las falencias del sistema, por ser capaces de ejercer su labor a pesar de enfrentarse a condiciones no favorables.

En este ejercicio reflexivo con relación a las debilidades del sistema público, las referencias específicas a secundaria se hicieron presentes. En los discursos aparecen con claridad las representaciones sobre primaria como un esquema con dificultades en la gestión y adaptación a la nueva realidad, pero que de todos modos "funciona" y, en ese sentido, es destacado el lugar de los maestros y el esquema organizacional de los centros educativos para esta propuesta. En cuanto a secundaria, las debilidades son resaltadas y prevalecen sobre las fortalezas; finalmente, con 
De Prácticas y discursos

Universidad Nacional del Nordeste

Centro de Estudios Sociales

Año 9, Número 14, 2020, octubre

ISSN 2250-6942
El lugar de las representaciones en los procesos de elección educativa.

Un análisis sobre las estrategias de los sectores medios montevideanos

The representation's role in the educational process choice. An analysis of the strategies of the montevideo middle class

relación a la formación terciaria, los entrevistados refieren generalmente a la Universidad de la República, y las representaciones sobre la misma están signadas por fortalezas y valoraciones altamente positivas.

\title{
LO PÚBLICO Y LOS VALORES
}

\author{
"Yo sé que en el pago me tienen idea \\ porque últimamente, toy fallando fiero, \\ yo que he sido siempre un buen militante, \\ tuve que tranzar con el mundo moderno. \\ A todos mis nietos los mando a un privado, \\ pero jamás nunca traicioné a Varela, \\ porque aunque se llame "High College Bilingüish" \\ adelante mío le dicen escuela". \\ Cuplé de los viejos militantes, \\ Agarrate Catalina (2008).
}

Amerita redactar un apartado nuevo para referir a los valores que la educación pública representa para las personas entrevistadas. Al momento de tener que enumerar las fortalezas de la enseñanza pública, surge en la gran mayoría de los discursos un relato sobre la importancia del carácter gratuito, democrático, integrador, de construcción de ciudadanía, que supone para las mismas este ámbito.

Es difícil analizar estas valoraciones sin evocar y sin pensar en la capacidad estructurante que en la configuración de estas representaciones tiene un pasado que da cuenta de una primacía estatal a nivel de la política, de una temprana y vertiginosa ampliación de la cobertura -a principios de siglo XX en primaria y desde mediados de siglo en secundaria- de una fuerte capitalización de la educación por parte de las clases medias y obreras como mecanismo de movilidad social ascendente y de una sociedad hiperintegrada asentada sobre el pilar de la educación.

Elegir la escuela pública se traduce en un relato identitario que marca pertenencias y que se constituye en una suerte de epifenómeno individual/colectivo que es parte y consecuencia de procesos de construcción social (Narodowski y Gottau, 2016). Esta práctica discursiva que es también acción de elección, este movimiento entre ideas y prácticas, habla de habitus compartidos en lo que concierne a la importancia de la educación en la trayecto- 
De Prácticas y discursos

Universidad Nacional del Nordeste

Centro de Estudios Sociales

Año 9, Número 14, 2020, Octubre

ISSN 2250-6942

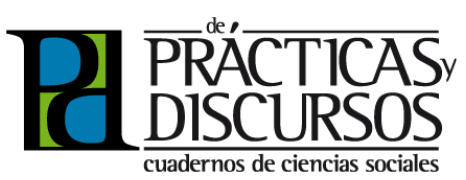

ria biográfica, pero también en lo que hace a la educación en su potencial integrador, en su capacidad de construir ciudadanía, en su carácter democratizante, en definitiva, en su relevancia a nivel social. Ser parte de la educación pública es pertenecer y participar de un espacio social más amplio que el propio centro educativo, es ser parte de lo público en la amplitud de su acepción.

Cierta tirantez en los discursos se identifica también entre la necesidad de proteger de las dificultades y carencias que tienen los liceos públicos y la intención de salvaguardar de "la burbuja" (expresión que se reitera en las entrevistas) o "micromundo" que representa lo privado. La respuesta a este dilema se traduce en la práctica, en la elección de lo privado por las fallas identificadas en el sistema público en determinado nivel y, a la vez, como respuesta a las necesidades de determinado momento de la etapa etaria de hijos e hijas.

no me gustaría que un hijo mío viviese siempre yendo a cuestiones privadas. Que haga, por ejemplo, jardín privado, escuela privada, liceo privado [...] porque es como un micromundo viste... no... no me gustaría. Claro, porque es como un micromundo, porque como que siempre está entre las personas que pueden pagar. (Entrevista 15)

La educación pública es resaltada en características y aspectos que igualmente hacen a la formación de las personas, cualidades que el ámbito privado no puede ofrecer porque son particularidades intrínsecas a lo público. Ser parte de la educación pública es rasgo distintivo de estos entrevistados de clase media, que muestran discursivamente no tener intenciones de ser parte del avance de una lógica promercado. Las tensiones están presentes entre las declaraciones de defensa a la enseñanza pública y la efectiva práctica de elección de un centro educativo privado. Tensión que se intenta aplacar esgrimiendo los motivos que llevaron a tal decisión, dando cuenta del estado de "declive institucional" que justifica migrar hacia lo privado.

Entonces, de alguna manera me confirmó que la opción que estábamos haciendo [con relación a la elección del liceo privado] no era en contra de la educación pública, porque yo en realidad soy producto de la educación pública, y ojalá hubiera estado en una posición de pensar que lo mejor que uno puede darle es el liceo público. (Entrevista 7) 
De Prácticas y discursos

Universidad Nacional del Nordeste

Centro de Estudios Sociales

Año 9, Número 14, 2020, 0ctubre

ISSN 2250-6942
El lugar de las representaciones en los procesos de elección educativa.

Un análisis sobre las estrategias de los sectores medios montevideanos

The representation's role in the educational process choice. An analysis of the strategies of the montevideo middle class

"Soy producto de la educación pública" es una frase que aparece en más de una entrevista $y$, podría decirse, es una frase socialmente acuñada en Uruguay. El conocimiento práctico que deviene de la experiencia de ser parte de la enseñanza pública es elemento estructurante de la percepción, del modo de sentir y pensar ese espacio, lo que hace a su representación y lo que estructura nuevas acciones, nuevas prácticas.

Es interesante destacar que surgen en algunos discursos expresiones que dan cuenta de cierta "resistencia" hacia lo privado y que provienen generalmente de quienes no tuvieron una experiencia propia de tránsito por ese sector. De todos modos, la tensión entre una alta estima por lo público-estatal y la práctica de optar por la oferta privada aparece en todos los discursos, en algunos de forma más explícita que en otros, como parte de un dilema entre el pensar, sentir y el hacer. El fragmento de entrevista citado a continuación es una muestra de cómo esa resistencia puede verse expresada desde el lenguaje, desde las palabras que utilizamos para nombrar las cosas. En este caso particularmente, se tuvo una corta experiencia de pasaje por lo privado en la escuela al momento de iniciarla.

le decíamos escuela, como el cuplé da la Catalina, los viejos militantes que le dicen escuela al colegio. Le decíamos escuela. Y al liceo también le decíamos liceo y no colegio. (Entrevista 17)

\section{VALORACIONES SOBRE LA OFERTA PRIVADA}

En Uruguay, la primacia estatal en materia de educación ha sido histórica y aún mantiene vigencia. Sin embargo, las propuestas de mercado vienen avanzando en forma paulatina e influyendo en la elección de ciertos sectores sociales desde hace unas décadas. Este proceso puede ser identificado en paises de la región y del resto de América Latina. En muchos de ellos con grados de avance más significativos que los que presenta Uruguay. Esto ha dado lugar a investigaciones sobre el tema, los que en general encuentran relación entre la estructura de clases y la elección escolar (Gamallo, 2011; Rojas y Falabella, 2013; Rojas, Falabella y Leyton, 2016; Narodowski y Gottau, 2016; Orellana, Caviedes, Bellei y Contreras, 2018). Este interés deviene justamente a partir del avance de las propuestas privadas que asumen a las familias como agentes con capacidad de optar por el centro educativo que más se adapte a sus necesidades, intereses y expectativas. 
De Prácticas y discursos

Universidad Nacional del Nordeste

Centro de Estudios Sociales

Año 9, Número 14, 2020, Octubre

ISSN 2250-6942

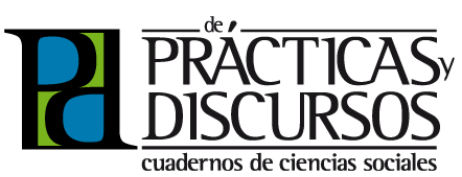

Algunas de las investigaciones referenciadas señalan que la elección escolar puede constituirse en un mecanismo de distinción de las clases medias, lo que puede ser interpretado como parte de un proceso más general de constitución de nuevas capas medias y de profunda reestructuración de los sistemas educacionales. Parten de la premisa de que las diferencias sociales se asocian a la desigual posesión de capitales (cultural, social, económico, escolar), cuyo juego en el campo educativo reproduce las diferencias de clase, al mismo tiempo que las legitima. En el proceso de elección, las clases medias llevan la ventaja por su mayor capital cultural, redes sociales de contacto y familiaridad con el sistema educativo (Orellana et al., 2018). Sin embargo, simultáneamente, se plantea que este fenómeno de variedad de oferta educativa que convierte a los referentes adultos de las familias en agentes de elección puede traducirse en un aumento de la individualización de los riesgos y un incremento de la responsabilidad que les corresponde a las familias en este asunto. En este sentido, se identifica un proceso de creciente incertidumbre y ansiedad que afecta mayormente a la clase media por ser justamente la que evalúa diferentes opciones (Orellana et al., 2018).

El recorrido para evaluar diferentes propuestas es algo que surge en las entrevistas y que da cuenta, en cierto modo, de lo que implica el proceso de elección para los referentes adultos. El mismo se transforma en la búsqueda de la mejor opción, de la que más se adapta a lo deseable, a lo entendido como necesario y que se ajusta a las posibilidades económicas del hogar.

Cabe destacar que los discursos no revelan que la opción por lo privado sea considerada con la finalidad de constituirse en un diferencial, en un elemento distintivo, sino que aparece más la idea de lo privado como una alternativa frente a la percepción de un sistema público que falla y que no es capaz de ofrecer las garantías de cuidado, control y seguimiento que los referentes adultos entienden necesarias. De hecho, esas son las principales fortalezas identificadas en lo privado, las que refieren a la gestión de los centros, a la vez que se explicita en general que en algunos aspectos no hay grandes diferencias con lo público porque se basan en la misma reglamentación, porque comparten los docentes:

Me parece que lo tenemos a veces sobrevalorado, como si tuviera un diferencial sustancial con lo público, que en realidad creo que desde el punto de vista de la formación y lo académico, realmente no sé si hay un diferencial. Lo que 
De Prácticas y discursos

Universidad Nacional del Nordeste

Centro de Estudios Sociales

Año 9, Número 14, 2020, Octubre

ISSN 2250-6942
El lugar de las representaciones en los procesos de elección educativa.

Un análisis sobre las estrategias de los sectores medios montevideanos

The representation's role in the educational process choice. An analysis of the strategies of the montevideo middle class

seguramente hay sí es un mayor espacio de contención de los chiquilines. (Entrevista 2)

"Los privados tienen un poco de eso, de ofrecer propuestas diferentes dentro del margen que les queda [...] Creo que los profesores son los mismos, y creo que hay muy buenos docentes en un ámbito y en otro. La diferencia está en la gestión y en una propuesta variada que ofrece lo privado que lo público no tiene. (Entrevista 11)

Las referencias al ausentismo docente en lo público y, por contraparte, la presencia de los profesores en el sector privado son un aspecto también mencionado en las entrevistas, identificados como debilidad de lo público y fortaleza de lo privado.

Es interesante que al preguntar por las fortalezas de la educación privada las respuestas se estructuran generalmente desde la comparación con el sector público. Este doble movimiento entre las valoraciones de lo privado y lo público, revela de algún modo que la opción por lo privado se explica fundamentalmente por lo público y que lo público no es desestimado con relación a lo privado. Secundaria pública en su nivel básico es desestimada en sí misma, como opción posible, dado el estado de declive institucional percibido por las personas entrevistadas.

Entre otros aspectos, aparecen como fortalezas las propuestas complementarias que ofrecen los centros del sector privado, surgiendo nuevamente la comparación con lo público. Este último es identificado con un déficit en lo que refiere a estas propuestas y con un grado de rigidez que lo limita en la posibilidad de salir de lo estrictamente curricular.

creo que tienen que tender a mejorar su calidad y su oferta educativa, porque de alguna manera digamos, viven de eso, es como una oferta que hacen, y la oferta tiene que estar actualizada, tiene que ser atractiva y tiene que tener resultados. Entonces, yo creo que eso es una ventaja. La escuela pública, o la educación pública, es como más rígida, está como encorsetada [...] Sin embargo, creo que el privado tiene como cierta libertad para poder tener una... digamos la oferta educativa básica, que tiene que ver con lo curricular, pero, sin embargo, le pueden agregar un montón de cosas, un montón de propuestas, y eso la hace ya... la hace no solo llamativa, sino que también creo que es necesario. (Entrevista 7) 
De Prácticas y discursos

Universidad Nacional del Nordeste

Centro de Estudios Sociales

Año 9, Número 14, 2020, Octubre

ISSN 2250-6942

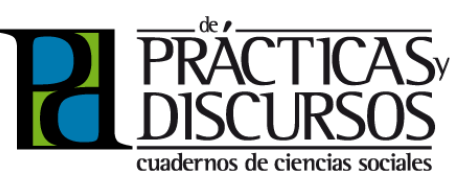

${ }^{12}$ El "batllismo" se vincula con la acción política de José Batlle y Ordóñez (político perteneciente al partido Colorado, nacido en 1856 y fallecido en 1929, dos veces presidente de la República entre 1903 y 1907 y entre 1911 y 1915) y del movimiento político que posibilitó y proyectó su influencia decisiva en la forja del Uruguay moderno (Caetano, 2010).
Por su parte, a la hora de referir a las debilidades, los entrevistados aluden a características del sector intrínsecas a la lógica de mercado. Si bien la flexibilidad es valorada con relación a la rigidez percibida en lo público, esa ductilidad es visualizada como riesgosa en tanto puede habilitar a que la propuesta sea moldeada en función de intereses no educativos, sino más bien de indole competitiva.

a veces tengo la sensación que hay colegios que son como... o sea... marketineros. Yo lo que hago es para que el padre esté contento, no en función de la educación. (Entrevista 3)

El avance de las propuestas de mercado en educación y la existencia de una variedad de alternativas puede conducir a los centros educativos a transitar por una lógica de captación de clientes, lo cual va en detrimento de la educación, según entienden las personas entrevistadas.

\section{CONSIDERACIONES FINALES}

Múltiples aspectos surgieron en los discursos que permitieron hilvanar y reconstruir en cierto modo la percepción, las formas de sentir, hacer y pensar de los entrevistados y las entrevistadas con relación al objeto de investigación. Es así que las valoraciones pro-Estado se fueron haciendo presentes en cada relato. La larga tradición estatal de Uruguay que encuentra su anclaje primario en la matriz social batllista ${ }^{12}$, seguramente incide en la percepción sobre el papel y la importancia del Estado, fundamentalmente, vinculada a la educación, la cual se constituye en una arena política de larga tradición pública que experimentó una temprana y vertiginosa ampliación de la cobertura, que fue altamente capitalizada por las clases medias y obreras como mecanismo de movilidad social ascendente y sobre la que se asentaron las bases para la conformación de una sociedad que pudo ser catalogada, pese a los cuestionamientos que cayeron sobre ella, como hiperintegrada. En este sentido, se recoge de cada discurso un relato acerca de la importancia del carácter gratuito, democrático, integrador, de construcción de ciudadanía, que supone para las personas entrevistadas este ámbito.

Esta experiencia discursiva, este movimiento entre ideas y prácticas, habla de habitus compartidos en lo que concierne a la 
importancia de la educación en la trayectoria biográfica, pero también en lo que hace a la educación en su potencial integrador, en su capacidad de construir ciudadanía, en su carácter democratizante, en definitiva, en su importancia a nivel social. La elección del centro educativo al que asistirán sus hijos adquiere en los entrevistados una significativa relevancia, y la opción por la escuela pública, en particular, se traduce en una suerte de epifenómeno individual/colectivo que es parte y consecuencia de procesos de construcción de ciudadanía social.

Como una marca identitaria, "soy producto de la educación pública" aparece de manera reiterada en las entrevistas y, podría decirse, es una frase socialmente acuñada en Uruguay. El conocimiento práctico que deviene de la experiencia de haber sido parte de la enseñanza pública es elemento estructurante de la percepción, del modo de sentir y pensar ese espacio, lo que hace a su representación y lo que estructura nuevas acciones y prácticas. Como expresión de este mismo proceso surgen los enunciados que dan cuenta de cierta "resistencia" hacia lo privado, sobre todo por parte de quienes no tuvieron una experiencia de tránsito por ese sector. Igualmente, es de destacar que la tensión entre una alta estima por lo público-estatal y la práctica de optar por la oferta privada aparece en todos los discursos, en algunos de forma más explícita que en otros, como parte de un dilema entre el pensar, sentir y el hacer.

Las tensiones están presentes entre las declaraciones de defensa a la enseñanza pública y la efectiva práctica de elección de un centro educativo privado. Tirantez que se intenta aplacar esgrimiendo los motivos que llevaron a tal decisión, dando cuenta del estado de "declive institucional" que justifica migrar hacia lo privado. Así, la referencia al estado del instrumento de reproducción, en este caso, el sistema educativo, aparece como la principal vía de argumentación para defender la elección por el mercado, pese a la gran estima y alta valoración de lo público-estatal, porque inevitablemente el juego discursivo trae elementos de justificación de aquellas acciones que parecen contradictorias. Esto se da, a su vez, y paradójicamente, bajo el contexto de gobiernos de coalición de izquierda en los que se otorgó, tanto a nivel discursivo como presupuestal, gran relevancia a la política educativa.

Las personas entrevistadas entienden que la educación pública tiene particularidades que le son intrínsecas, que hacen a la formación de las personas en un sentido amplio, dados los valores 
De Prácticas y discursos

Universidad Nacional del Nordeste

Centro de Estudios Sociales

Año 9, Número 14, 2020, Octubre

ISSN 2250-6942

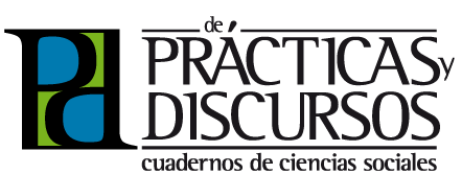

democráticos que pueden incorporarse al ser parte de aulas que están integradas por estudiantes provenientes de diferentes realidades socioeconómicas. En líneas generales, las personas entrevistadas muestran discursivamente no tener intenciones de ser parte del avance de una lógica promercado. Tampoco demuestran que el interés en optar por el sector privado esté dado por los posibles contactos que se puedan generar al vincularse con personas de un estatus socioeconómico más elevado, es decir, por la intención en incrementar el capital social de sus hijos e hijas.

De todos modos, vale la pena reconocer que, aun teniendo la posibilidad, estos referentes adultos de clase media decidieron optar por lo público y no migrar hacia lo privado al elegir la escuela. Fueron considerados aspectos de carácter ideológicofilosófico en ese proceso de elección. La representación erigida sobre la educación pública está asociada a conceptos tales como construcción de ciudadanía, universalismo, igualdad, convivencia e integración. Y todo esto puede encontrarse en la matriz social uruguaya sobre la que se construyó un sistema educativo que tempranamente buscó no ser excluyente. La valoración y estima de lo público-estatal por parte de los sectores medios es algo que atañe con particularidad a esta arena política. Esto es un aspecto no menor a la hora de pensar la gestión de la política educativa y de buscar hacer frente al proceso de segregación que la afecta; en tanto es factible revertir los procesos de migración hacia el sector privado volviendo a captar a estudiantes provenientes de los sectores medios, favoreciendo, de este modo, la consolidación de procesos de integración social.

Las tensiones y oscilaciones dan cuenta de una práctica discursiva que revela la complejidad de la propia práctica de elección. Lo privado no aparece como una opción per se. Y esto, de algún modo, condice con la postura pro-Estado que cada entrevistado deja entrever en el plano discursivo. Que puedan identificarse dentro de las clases medias posturas con esta orientación da cuenta de algún modo de la existencia de una especie de reservorio, que guarda estrecha relación con la configuración de la matriz social uruguaya, con el legado de orientaciones de politica en las que el Estado ejerció un papel central y con la participación que las clases medias tuvieron en ese contexto. El "país de las medianías" y el de la "hiperintegración" se ven conjugados y materializados en esa imagen que transmite la popular frase de Varela: "los que una vez se han encontrado juntos en los 
De Prácticas y discursos

Universidad Nacional del Nordeste

Centro de Estudios Sociales

Año 9, Número 14, 2020, octubre

ISSN 2250-6942
El lugar de las representaciones en los procesos de elección educativa.

Un análisis sobre las estrategias de los sectores medios montevideanos

The representation's role in the educational process choice. An analysis of the strategies of the montevideo middle class

bancos de una escuela, a la que concurren haciendo uso de un mismo derecho, se acostumbran a considerarse iguales". Algo de estas ideas queda evidenciado en los relatos que trae esta investigación, y refleja un modo de sentir y pensar particular. Pese al avance privatizador que se viene dando en Uruguay en materia de educación, en comparación con otros países del continente resulta ser un desarrollo menor. Ello no quita, igualmente, que sea necesario y urgente prestarle atención, ya que esta suerte de reservorio propúblico al que se hacia referencia, puede con el tiempo y con el avance de este tipo de procesos, ir diluyéndose y perdiendo fuerza. Esto resulta aún más preocupante en un contexto que tiende a resignificar lo público, discursivamente y en términos presupuestales. Tal como señalan Katzman y Retamoso (2007), las formas típicas de interacción entre las clases suelen ser el fruto de lentos procesos de decantación de raices históricas profundas. A lo largo de estos, y al impulso de transformaciones en distintas esferas, las ideas y representaciones estructuradas sobre la equidad, la justicia y las formas de integración social originalmente incorporadas en las matrices socioculturales nacionales se consolidan o se modifican al influjo de nuevas circunstancias.

\section{BIBLIOGRAFÍA}

BELLEI, C. (2013). El estudio de la segregación socioeconómica y académica de la educación chilena. Estudios Pedagógicos, (1)39, 325-345. Recuperado de 11 de octubre de 2018 de: http:// www.scielo.cl/pdf/estped/v39n1/art19.pdf

BOURDIEU, P. (1988). La distinción. Criterio y bases del gusto. Madrid, España: Taurus.

(2007). Los tres estados del capital cultural. En Bourdieu Pierre. Campo del poder y reproducción social. Colección Enjeux (pp. 195-202). Córdoba: Ferreyra Editor.

(2011). Las estrategias de reproducción social. Buenos Aires, Argentina: Siglo XXI Editores.

BOURDIEU, P. Y PASSERON, J.C. (1977). La reproducción. Elementos para una teoría de sistema de enseñanza. Barcelona, España: Laia. 
CAETANO, G. (2010). Ciudadanía y Nación en el Uruguay del Centenario (1910-1930). La forja de una cultura estatista. Revista Iberoamericana, 39, 161-176.

DELGADO, J.M. Y GUTIÉRREZ, J. (COORD.) (1999). Métodos y técnicas cualitativas de investigación en Ciencias Sociales. Madrid, España: Sintesis.

DUBET, F. (2007). El declive y las mutaciones de la institución. Revista de antropología social, 16, 39-66.

GAMALLO, G. (2011). Mercantilización del bienestar. Hogares pobres y escuelas privadas. Revista de Instituciones, Ideas y Mercados, 55, 189-233.

GARCÉ, A. Y YAFFÉ, J. (2004). La era progresista. Montevideo: Fin de Siglo.

KAZTMAN, R. Y RETAMOSO, A. (2006). Segregación residencial en Montevideo: Desafios para la equidad educativa. Colección Monitor social, (7).

MARTíN CRIADO, E. (2014). Mentiras, inconsistencias y ambivalencias. Teoría de la acción y análisis de discurso. Revista Internacional de Sociología, (72), 115-138.

NARODOWSKI, M. Y GOTTAU, V. (2017). Clases medias y escuela pública. La elección escolar como resistencia. Perfiles educativos, 39(157), 34-51.

ORELLANA, V.; CAVIEDES, S.; BELLEI, C. Y CONTRERAS, M. (2018). La elección de escuela como fenómeno sociológico. Una revisión de literatura. Revista Brasileira de Educação, 23, 1-19.

RAVEAUD, M. Y VAN ZANTEN, A. (2006). Choosing the local school: Middle class parents' values and social and ethnic mix in London and Paris. Journal of Education Policy, 22, 1, 107-124.

REIMERS, F. (2000). Educación, desigualdad y opciones de política en América Latina en el siglo XXI. Revista Latinoamericana de Estudios Educativos, (XXX)2. 
De Prácticas y discursos

Universidad Nacional del Nordeste

Centro de Estudios Sociales

Año 9, Número 14, 2020, octubre

ISSN 2250-6942
El lugar de las representaciones en los procesos de elección educativa. Un análisis sobre las estrategias de los sectores medios montevideanos

The representation's role in the educational process choice. An analysis of the strategies of the montevideo middle class

ROJAS, M.T. Y FALABELLA, A. (2013). Estrategias educativas de las clases medias: opciones y dilemas. En La frágil Clase Media: estudios sobre grupos medios en el Chile contemporáneo. Santiago de Chile, Chile: Uredes.

ROJAS, M.T.; FALABELLA, A. Y LEYTON, D. (2016). Madres de clases medias frente al mercado educativo en Chile: decisiones y dilemas. En Corvalán, J. Carrasco, A. y García Huidobro, J.E. (eds.) Mercado escolar y oportunidad educacional: libertad, diversidad y desigualdad (231-265). Santiago: Ediciones UC.

VALLES, M. (2000) Técnicas cualitativas de investigación social. Reflexión metodológica y práctica profesional. Madrid, España: Sintesis.

VAN ZANTEN, A. (2007). Reflexividad y elección de la escuela por los padres de la clase media en Francia. Revista de Antropología Social, 16, 245-277. 